\title{
Soluble Polyimides Based on Alkyldiaminobenzophenone
}

\author{
Yusuke TsudA, Takehiro KaWAUCHI, Norihiko HryoshI, ${ }^{*}$ and Shuntaro MATAKA** \\ Department of Biochemistry \& Applied Chemistry, Kurume National College of Technology, \\ 1-1-1 Komorino-machi, Kurume, Fukuoka 830-8555, Japan \\ *Advanced Engineering School, Kurume National College of Technology, \\ 1-1-1 Komorino-machi, Kurume, Fukuoka 830-8555, Japan \\ ** Institute of Advanced Material, Kyushu University, \\ 6-1 Kasuga-Koh-en, Kasuga-shi, Fukuoka 816-8580, Japan
}

(Received January 17, 2000)

\begin{abstract}
The synthesis and characterization of a novel series of soluble polyimides based on alkyldiaminobenzophenone (ADBP) having long chain linear alkyl groups with 9-14 carbon atoms are described. Polyimides containing tetracarboxylic dianhydrides such as 3,3',4,4'-benzophenonetetracarboxylic dianhydride (BTDA) and ADBP with even number carbon atoms were soluble in polar solvents such as $N$-methyl-2-pyrrolidone (NMP), while polyimides containing BTDA and ADBP with odd number carbon atoms (ADBP-9, 11, 13) were insoluble in NMP. These unique odd-even effects of solubility were assumed due to the conformational effects of long chain linear alkyl groups. Soluble copolyimides based on ADBP with conventional aromatic diamine, diaminodiphenylether (DDE) can be obtained. The incorporation of DDE resulted in increment of molecular weight and improve the thermal stability. Soluble polyimides and copolyimides based on ADBP were soluble in various polar solvents and exhibited high thermal stability in air and nitrogen.

KEY WORDS Polyimide / Soluble Polyimide / Alkyldiaminobenzophenone / Odd-Even Effect / Copolymerization / Thermal Stability / Solubility /
\end{abstract}

Polyimides exhibit excellent thermal and mechanical properties and have extensive engineering and microelectronics applications. ${ }^{1}$ Aromatic polyimides such as pyromellitic polyimides are prepared from aromatic diamines and aromatic tetracarboxylic dianhydrides via poly(amic acid)s. Since conventional aromatic polyimides are insoluble, these polymers are usually processed as the corresponding soluble poly(amic acid) precursors and then either thermally or chemically imidized. However, there are some problems owing to the unstability of poly(amic acid)s and liberation of water in imidization process. Therefore, solvent soluble polyimides processable without difficulty are desired. Several approaches to improve the solubility of the polyimides have been investigated. $^{2-28}$ The solubility of polyimides has been successfully improved by the incorporation of fluorine moieties, ${ }^{2-7}$ or chlorine moieties, ${ }^{8}$ or bulky side groups, ${ }^{9-11}$ or pendant phenyl groups, ${ }^{12-16}$ or pendant benzoxazole or benzothiazole groups, ${ }^{17}$ or polydimethylsiloxane segment, ${ }^{18,19}$ or polyalicyclic structures, ${ }^{20-25}$ or naphthalene moieties, ${ }^{26}$ or spiro structure, ${ }^{27}$ or pyrazoline moieties ${ }^{28}$ or benzhydrol unit ${ }^{29}$ into the polymer backbone. Recently the authors reported the synthesis and characterization of soluble polyimides and copolyimides prepared from a polyalicyclic tetracarboxylic dianhydride, 2,3,5-tricarboxycyclopentyl acetic dianhydride (TCA-AH) and aromatic diamines. ${ }^{30,31}$

This paper reports the synthesis and characterization of soluble polyimides based on alkyldiaminobenzophenone (ADBP) having long chain linear alkyl groups with 9-14 carbon atoms and conventional tetracarboxylic dianhydrides such as 3,3',4,4'-benzophenonetetracarboxylic dianhydride (BTDA). Factors affecting the solubility of final polymers such as alkyl chain length of ADBP, variation in tetracarboxylic dianhydrides and copolymerization effects using conventional aromatic dia- mine, 4,4'-diaminodiphenylether (DDE) together with ADBP are also considered in some detail. The thermal stability of obtained polyimides and copolyimides are characterized with thermogravimetric analysis (TGA) and the solubility of these polymers was determined with various common solvents. The attachment of alkyl side chains to polyimides has recently been used to increase pretilt angles generated by the polymers in alignment layer applications in liquid crystal displays (LCDs) and thus these studies are practically of great importance. ${ }^{32-34}$

\section{EXPERIMENTAL}

\section{Materials}

3,5-Dinitrobenzoyl chloride and $n$-alkylbenzenes were purchased from Tokyo Chemical Industry Co., Ltd. and used as received. Aluminium chloride, nitrobenzene, and $10 \% \mathrm{Pd} / \mathrm{C}$ were purchased from Wako Pure Chemical Industries, Ltd. and used as received. Hydrogen gas (Kyushu Fuel Co., Ltd.) was used as received. TCA-AH ( $\mathrm{mp}$ $194-195^{\circ} \mathrm{C}$ from acetic anhydride) was kindly supplied by JSR Corporation. Pyrromellitic dianhydride (PMDA) (Mitsubishi Gas Chemical), BTDA (Mitsui Toatsu Chemicals Inc.), BPDA (Ube Industries, Ltd.), and 4,4'diaminodiphenylether (DDE) (Wakayama Seika Kogyo Co., Ltd.) were used as received. $N$-methyl-2-pyrrolidone (NMP) (Mitsubishi Kasei) were distilled under reduced pressure. Reagent grade $N, N$-dimethylformamide (DMF), $N, N$-dimethylacetamide (DMAc), dimethyl sulfoxide (DMSO), 1,3-dimethyl-2-imidazolidinone (DMI), $m$-cresol, tetrahydrofuran (THF), dichloromethane, sulfuric acid, acetic anhydride, and pyridine were purchased from Katayama Chemical Industries Co., Ltd. and used as received. Dimethyl sulfoxide- $d_{6}$ (DMSO- $d_{6}$ ) and pyridine- $d_{5}$ (Aldrich Chem. Co.), and other conven- 
tional reagents were used as received.

\section{Measurement}

Inherent viscosities of all polymers were measured using Cannon Fenske viscometers at a concentration of 0.5 $\mathrm{g} \mathrm{dL}^{-1}$ in NMP at $30^{\circ} \mathrm{C}$. Thermogravimetric analysis (TGA) was performed on a Shimadzu thermogravimetric analyzer Model TGA-50 in air or nitrogen at a heating rate of $10^{\circ} \mathrm{C} \mathrm{min}^{-1} .{ }^{1} \mathrm{H}$ NMR spectra were measured on a JEOL JNM-EX $270 \mathrm{FT}$ NMR in a DMSO- $d_{6}$ with tetramethylsilane (TMS) as internal reference. IR spectra were measured on a JASCO IR Report-100 spectrophotometer.

\section{Synthesis of 3,5-Dinitro-4'-n-dodecylbenzophenone}

3,5-Dinitrobenzoyl chloride $(7.48 \mathrm{~g}, 32.4 \mathrm{mmol})$ and $n$ dodecylbenzene $(8 \mathrm{~g}, 32.4 \mathrm{mmol})$ were dissolved in 200 $\mathrm{mL}$ nitrobenzene. The resulting solution was cooled to below $10^{\circ} \mathrm{C}$ in an ice-water bath after which $6.49 \mathrm{~g}(48.7$ mmol) of anhydrous $\mathrm{AlCl}_{3}$ were added slowly and in small portions so that the reaction temperature would not rise above $15^{\circ} \mathrm{C}$. After all the $\mathrm{AlCl}_{3}$ had been added, the ice bath was removed and the reaction mixture was stirred at room temperature so that the suspension became clear solution, and kept stirring at $80^{\circ} \mathrm{C}$ for $3 \mathrm{~h}$. The reaction mixture was then poured into a mixture of $25 \mathrm{~mL}$ concentrated $\mathrm{HCl}$ and $100 \mathrm{~mL}$ ice-water. The organic layer was separated and washed sequentially with $\mathrm{H}_{2} \mathrm{O}, 10 \%$ aqueous $\mathrm{NaOH}$, and $\mathrm{H}_{2} \mathrm{O}$, and dried over anhydrous sodium sulfate, filtered and the nitrobenzene was removed under vacuum distillation. The obtained solid was recrystallized from ethanol with charcoal, to give $8.50 \mathrm{~g}$ (19.3 mmol, yield $60 \%)$ of white crystals. Mp $56.5-57.8^{\circ} \mathrm{C} ;{ }^{1} \mathrm{H}$ NMR (DMSO- $\left.d_{6}\right): \delta 0.85$ (t, $3 \mathrm{H},-\mathrm{Ar}^{-}$ $\left.\mathrm{CH}_{2}-\mathrm{CH}_{2}-\left(\mathrm{CH}_{2}\right)_{9}-\mathrm{CH}_{3}, J=6.59 \mathrm{~Hz}\right), 1.24(\mathrm{~m}, 18 \mathrm{H},-\mathrm{Ar}-$ $\left.\mathrm{CH}_{2}-\mathrm{CH}_{2}-\left(\mathrm{CH}_{2}\right)_{9}-\mathrm{CH}_{3}\right), 1.62\left(\mathrm{~m}, 2 \mathrm{H},-\mathrm{Ar}-\mathrm{CH}_{2}-\mathrm{CH}_{2}-\right.$ $\left.\left(\mathrm{CH}_{2}\right)_{9}-\mathrm{CH}_{3}\right), 2.70$ (t, 2H,-Ar- $\mathrm{CH}_{2}-\mathrm{CH}_{2}-\left(\mathrm{CH}_{2}\right)_{9}-\mathrm{CH}_{3}, \mathrm{~J}$ $=7.59 \mathrm{~Hz}), 7.44(\mathrm{~d}, 2 \mathrm{H}$, ortho to $n$-dodecyl group, $J=7.92$ $\mathrm{Hz}$ ) 7.79 (d, 2H, meta to $n$-dodecyl group, $J=7.92 \mathrm{~Hz}$ ), $8.77(\mathrm{~s}, 2 \mathrm{H}$, ortho to $\mathrm{C}=\mathrm{O})$, and $9.04 \mathrm{ppm}(\mathrm{s}, 1 \mathrm{H}$ para to $\mathrm{C}=\mathrm{O})$; IR $(\mathrm{KBr}): 1645(\mathrm{C}=\mathrm{O}), 1530,1335\left(\mathrm{NO}_{2}\right) \mathrm{cm}^{-1}$. Anal. Calcd for $\mathrm{C}_{25} \mathrm{H}_{32} \mathrm{~N}_{2} \mathrm{O}_{5}: \mathrm{C}, 68.16 \% ; \mathrm{H}, 7.32 \% ; \mathrm{N}$, $6.36 \%$. Found: C, $68.29 \%$; H, 7.29\%; N, 6.40\%.

Other 4- $n$-alkyl-3',5'-dinitrobenzophenones were synthesized as above (yield $50-70 \%$ ). Melting point (mp), ${ }^{1} \mathrm{H}$ NMR, IR and elemental analysis are as follows.

\section{3,5-Dinitro-4'-nonylbenzophenone}

Mp 64.5-65.0 ${ }^{\circ}{ }^{1}{ }^{1} \mathrm{H}$ NMR (DMSO- $\left.d_{6}\right): \delta 0.85(\mathrm{t}, 3 \mathrm{H}$, $\left.\mathrm{Ar}-\mathrm{CH}_{2}-\mathrm{CH}_{2}-\left(\mathrm{CH}_{2}\right)_{9}-\mathrm{CH}_{3}, J=6.59 \mathrm{~Hz}\right), 1.24(\mathrm{~m}, 12 \mathrm{H}$, $\left.-\mathrm{Ar}-\mathrm{CH}_{2}-\mathrm{CH}_{2}-\left(\mathrm{CH}_{2}\right)_{6}-\mathrm{CH}_{3}\right), 1.62\left(\mathrm{~m}, 2 \mathrm{H},-\mathrm{Ar}-\mathrm{CH}_{2}-\mathrm{CH}_{2}\right.$ $\left.-\left(\mathrm{CH}_{2}\right)_{6}-\mathrm{CH}_{3}\right), 2.70\left(\mathbf{t}, 2 \mathrm{H},-\mathrm{Ar}-\mathrm{CH}_{2}-\mathrm{CH}_{2}-\left(\mathrm{CH}_{2}\right)_{6}-\mathrm{CH}_{3}, \mathrm{~J}\right.$ $=7.59 \mathrm{~Hz}), 7.43(\delta, 2 \mathrm{H}$, ortho to $n$-nonyl group, $J=8.25$ $\mathrm{Hz}), 7.78(\mathrm{~d}, 2 \mathrm{H}$, meta to $n$-nonyl group, $J=8.25 \mathrm{~Hz}$ ), $8.76(\mathrm{~s}, 2 \mathrm{H}$, ortho to $\mathrm{C}=\mathrm{O}), 9.03(\mathrm{~s}, 1 \mathrm{H}$ para to $\mathrm{C}=\mathrm{O})$ ppm; IR (KBr): $1655(\mathrm{C}=\mathrm{O}), 1540,1340\left(\mathrm{NO}_{2}\right) \mathrm{cm}^{-1}$. Anal. Calcd for $\mathrm{C}_{22} \mathrm{H}_{26} \mathrm{~N}_{2} \mathrm{O}_{5}: \mathrm{C}, 66.32 \% ; \mathrm{H}, 6.58 \% ; \mathrm{N}$, 7.03\%. Found: C, $66.36 \% ; \mathrm{H}, 6.57 \%, \mathrm{~N} ; 7.04 \%$.

\section{3,5-Dinitro-4'-decylbenzophenone}

Mp 55.0-56.1 ${ }^{\circ} \mathrm{C} ;{ }^{1} \mathrm{H}$ NMR (DMSO- $d_{6}$ ): $\delta 0.85(\mathrm{t}, 3 \mathrm{H}$, $\left.-\mathrm{Ar}-\mathrm{CH}_{2}-\mathrm{CH}_{2}-\left(\mathrm{CH}_{2}\right)_{7}-\mathrm{CH}_{3}, J=6.59 \mathrm{~Hz}\right), 1.23(\mathrm{~m}, 14 \mathrm{H}$, $\left.-\mathrm{Ar}-\mathrm{CH}_{2}-\mathrm{CH}_{2}-\left(\mathrm{CH}_{2}\right)_{7}-\mathrm{CH}_{3}\right), 1.62\left(\mathrm{~m}, 2 \mathrm{H},-\mathrm{Ar}-\mathrm{CH}_{2}-\mathrm{CH}_{2}-\right.$
$\left.\left(\mathrm{CH}_{2}\right)_{7}-\mathrm{CH}_{3}\right), 2.70$ (t, 2H,-Ar- $\mathrm{CH}_{2}-\mathrm{CH}_{2}-\left(\mathrm{CH}_{2}\right)_{7}-\mathrm{CH}_{3}, J=$ $7.59 \mathrm{~Hz}$ ), $7.45(\mathrm{~d}, 2 \mathrm{H}$, ortho to $n$-decyl group, $J=8.25$ $\mathrm{Hz}), 7.80(\mathrm{~d}, 2 \mathrm{H}$, meta to $n$-decyl group, $J=8.25 \mathrm{~Hz}), 8.77$ $(\mathrm{s}, 2 \mathrm{H}$, ortho to $\mathrm{C}=\mathrm{O}), 9.04(\mathrm{~s}, 1 \mathrm{H}$, para to $\mathrm{C}=\mathrm{O}) \mathrm{ppm}$. IR $(\mathrm{KBr}): 1650(\mathrm{C}=\mathrm{O}), 1540,1340\left(\mathrm{NO}_{2}\right) \mathrm{cm}^{-1}$. Anal. Calcd for $\mathrm{C}_{23} \mathrm{H}_{28} \mathrm{~N}_{2} \mathrm{O}_{5}: \mathrm{C}, 66.97 \% ; \mathrm{H}, 6.84 \% ; \mathrm{N}, 6.79 \%$. Found: C, $66.58 \%$; H, 6.27\%; N, 7.28\%.

\section{3,5-Dinitro-4'-undecylbenzophenone}

Mp 55.3-56.3 ${ }^{\circ} \mathrm{C} ;{ }^{1} \mathrm{H}$ NMR (DMSO- $\left.d_{6}\right): \delta 0.85(\mathrm{t}, 3 \mathrm{H}$, $\left.-\mathrm{Ar}-\mathrm{CH}_{2}-\mathrm{CH}_{2}-\left(\mathrm{CH}_{2}\right)_{8}-\mathrm{CH}_{3}, J=6.59 \mathrm{~Hz}\right), 1.24(\mathrm{~m}, 16 \mathrm{H}$, $\left.-\mathrm{Ar}-\mathrm{CH}_{2}-\mathrm{CH}_{2}-\left(\mathrm{CH}_{2}\right)_{8}-\mathrm{CH}_{3}\right), 1.62\left(\mathrm{~m}, 2 \mathrm{H},-\mathrm{Ar}-\mathrm{CH}_{2}-\mathrm{CH}_{2}{ }^{-}\right.$ $\left.\left(\mathrm{CH}_{2}\right)_{8}-\mathrm{CH}_{3}\right), 2.70$ (t, $2 \mathrm{H},-\mathrm{Ar}-\mathrm{CH}_{2}-\mathrm{CH}_{2}-\left(\mathrm{CH}_{2}\right)_{8}-\mathrm{CH}_{3}, \mathrm{~J}=$ $7.59 \mathrm{~Hz}), 7.43(\mathrm{~d}, 2 \mathrm{H}$, ortho to $n$-undecyl group, $J=8.25$ $\mathrm{Hz}), 7.79(\mathrm{~d}, 2 \mathrm{H}$, meta to $n$-undecyl group, $J=8.25 \mathrm{~Hz}$ ), $8.77(\mathrm{~s}, 2 \mathrm{H}$, ortho to $\mathrm{C}=\mathrm{O}), 9.04(\mathrm{~s}, 1 \mathrm{H}$ para to $\mathrm{C}=\mathrm{O})$ ppm. IR (KBr): $1655(\mathrm{C}=\mathrm{O}), 1540,1340\left(\mathrm{NO}_{2}\right) \mathrm{cm}^{-1}$. Anal. Calcd for $\mathrm{C}_{26} \mathrm{H}_{30} \mathrm{~N}_{2} \mathrm{O}_{5}: \mathrm{C}, 67.59 \% ; \mathrm{H}, 7.09 \% ; \mathrm{N}$, $6.57 \%$. Found: C, $67.62 \%$;, $7.11 \%$; N $6.60 \%$.

\section{3,5-Dinitro-4'-tridecylbenzophenone}

Mp 53.0-54.2 ${ }^{\circ} \mathrm{C} ;{ }^{1} \mathrm{H}$ NMR (DMSO- $d_{6}$ ): $\delta 0.85(\mathrm{t}, 3 \mathrm{H}$, $\left.-\mathrm{Ar}-\mathrm{CH}_{2}-\mathrm{CH}_{2}-\left(\mathrm{CH}_{2}\right)_{10}-\mathrm{CH}_{3}, J=6.60 \mathrm{~Hz}\right), 1.23(\mathrm{~m}, 20 \mathrm{H}$, $\left.-\mathrm{Ar}-\mathrm{CH}_{2}-\mathrm{CH}_{2}-\left(\mathrm{CH}_{2}\right)_{10}-\mathrm{CH}_{3}\right), 1.62\left(\mathrm{~m}, 2 \mathrm{H},-\mathrm{Ar}-\mathrm{CH}_{2}-\mathrm{CH}_{2}\right.$ $\left.-\left(\mathrm{CH}_{2}\right)_{10}-\mathrm{CH}_{3}\right), 2.70$ (t, 2H,- $\mathrm{Ar}-\mathrm{CH}_{2}-\mathrm{CH}_{2}-\left(\mathrm{CH}_{2}\right)_{10}-\mathrm{CH}_{3}$, $J=7.59 \mathrm{~Hz}), 7.44$ (d, $2 \mathrm{H}$, ortho to $n$-tridecyl group, $J=$ $8.25 \mathrm{~Hz}), 7.79(\mathrm{~d}, 2 \mathrm{H}$, meta to $n$-tridecyl group, $J=8.25$ $\mathrm{Hz}), 8.77(\mathrm{~s}, 2 \mathrm{H}$, ortho to $\mathrm{C}=\mathrm{O}), 9.04(\mathrm{~s}, 1 \mathrm{H}$ para to $\mathrm{C}=$ O) ppm. IR (KBr): $1655(\mathrm{C}=\mathrm{O}), 1530,1340\left(\mathrm{NO}_{2}\right) \mathrm{cm}^{-1}$. Anal. Calcd for $\mathrm{C}_{25} \mathrm{H}_{34} \mathrm{~N}_{2} \mathrm{O}_{5}: \mathrm{C}, 68.70 \% ; \mathrm{H}, 7.54 \% ; \mathrm{N}$, $6.16 \%$. Found: C, $69.13 \% ; \mathrm{H}, 7.61 \% ; \mathrm{N}, 6.03 \%$.

\section{3,5-Dinitro-4'-teradecylbenzophenone}

Mp 52.0-53.0 ${ }^{\circ} \mathrm{C} ;{ }^{1} \mathrm{H}$ NMR (DMSO- $\left.d_{6}\right): \delta 0.84(\mathrm{t}, 3 \mathrm{H},-$ Ar $\left.\mathrm{CH}_{2}-\mathrm{CH}_{2}-\left(\mathrm{CH}_{2}\right)_{11}-\mathrm{CH}_{3}, J=6.27 \mathrm{~Hz}\right), 1.22(\mathrm{~m}, 22 \mathrm{H}$, $\left.\mathrm{Ar}-\mathrm{CH}_{2}-\mathrm{CH}_{2}-\left(\mathrm{CH}_{2}\right)_{11}-\mathrm{CH}_{3}\right), 1.60\left(\mathrm{~m}, 2 \mathrm{H},-\mathrm{Ar}-\mathrm{CH}_{2}-\mathrm{CH}_{2}-\right.$ $\left.\left(\mathrm{CH}_{2}\right)_{11}-\mathrm{CH}_{3}\right), 2.68\left(\mathrm{t}, 2 \mathrm{H},-\mathrm{Ar}-\mathrm{CH}_{2}-\mathrm{CH}_{2}-\left(\mathrm{CH}_{2}\right)_{9}-\mathrm{CH}_{3}, \mathrm{~J}\right.$ $=7.59 \mathrm{~Hz}), 7.43(\mathrm{~d}, 2 \mathrm{H}$, ortho to $n$-tetradecyl group, $J=$ $8.25 \mathrm{~Hz}), 7.79(\mathrm{~d}, 2 \mathrm{H}$, meta to $n$-dodecyl group, $J=8.25$ $\mathrm{Hz}), 8.76(\mathrm{~s}, 2 \mathrm{H}$, ortho to $\mathrm{C}=\mathrm{O}), 9.04(\mathrm{~s}, 1 \mathrm{H}$ para to $\mathrm{C}=$ O) ppm; IR (KBr): $1655(\mathrm{C}=\mathrm{O}), 1540,1340\left(\mathrm{NO}_{2}\right) \mathrm{cm}^{-1}$. Anal. Calcd for $\mathrm{C}_{27} \mathrm{H}_{36} \mathrm{~N}_{2} \mathrm{O}_{5}: \mathrm{C}, 69.21 \% ; \mathrm{H}, 7.74 \% ; \mathrm{N}$, $5.98 \%$. Found: C, $69.34 \%$; H, 7.75\%; N, 5.95\%.

\section{Synthesis of 3,5-Diamino-4'-n-dodecylbenzophenone ( $A D$ $B P-12$ )}

A mixture of $8 \mathrm{~g}(18.16 \mathrm{mmol})$ of 3,5 -diamino- $4^{\prime}-n$ dodecylbenzophenone and $1.82 \mathrm{~g}$ of $10 \%$ palladium on carbon $(\mathrm{Pd} / \mathrm{C})$ in $200 \mathrm{~mL}$ of $\mathrm{DMF}$ was stirred at $80^{\circ} \mathrm{C}$ for $12 \mathrm{~h}$ using the apparatus attached with a balloon filled with hydrogen gas. The solution was filtered and DMF was evaporated. The obtained crude diamine was recrystallized from ethanol/water mixture with charcoal to give $3.50 \mathrm{~g}$ (yield $51 \%$ ) of pale brown powder. $\mathrm{Mp} 67.0-$ $68.0^{\circ} \mathrm{C} ;{ }^{1} \mathrm{H}$ NMR (DMSO- $d_{6}$ ): $\delta 0.85\left(\mathrm{t}, 3 \mathrm{H},-\mathrm{Ar}-\mathrm{CH}_{2}-\mathrm{CH}_{2}\right.$ $\left.-\left(\mathrm{CH}_{2}\right)_{9}-\mathrm{CH}_{3}, J=6.59 \mathrm{~Hz}\right), 1.23\left(\mathrm{~m}, 18 \mathrm{H},-\mathrm{Ar}-\mathrm{CH}_{2}-\mathrm{CH}_{2}-\right.$ $\left.\left(\mathrm{CH}_{2}\right)_{9}-\mathrm{CH}_{3}\right), \quad 1.59\left(\mathrm{~m}, 2 \mathrm{H},-\mathrm{Ar}-\mathrm{CH}_{2}-\mathrm{CH}_{2}-\left(\mathrm{CH}_{2}\right)_{9}-\mathrm{CH}_{3}\right)$, 2.64(t, $\left.2 \mathrm{H},-\mathrm{Ar}-\mathrm{CH}_{2}-\mathrm{CH}_{2}-\left(\mathrm{CH}_{2}\right)_{9}-\mathrm{CH}_{3}, J=7.26 \mathrm{~Hz}\right), 5.00$ $\left(\mathrm{s}, 4 \mathrm{H}, \mathrm{NH}_{2}\right), 6.07(\mathrm{t}, 1 \mathrm{H}$, para to $\mathrm{C}=\mathrm{O}, J=1.98 \mathrm{~Hz}), 6.14$ (d, $2 \mathrm{H}$, ortho to $\mathrm{C}=\mathrm{O}, J=1.98 \mathrm{~Hz}), 7.32(\mathrm{~d}, 2 \mathrm{H}$, ortho to $n$-dodecyl group, $J=7.59 \mathrm{~Hz}), 7.62(\mathrm{~d}, 2 \mathrm{H}$, meta to $n$ dodecyl group, $J=7.59 \mathrm{~Hz}) \mathrm{ppm}$. Detailed description of ${ }^{1} \mathrm{H}$ NMR spectra is given in the next section. IR ( $\mathrm{KBr}$ ): 
$3350\left(\mathrm{NH}_{2}\right), 1635(\mathrm{C}=\mathrm{O}) \mathrm{cm}^{-1}$. Anal. Calcd for $\mathrm{C}_{25} \mathrm{H}_{36} \mathrm{~N}_{2}$ O: C, $78.90 \%$; H, 9.53\%; N, 7.36\%. Found: C, 78.92\%; H, $9.49 \%$; N, $7.27 \%$. Other 4- $n$-alkyl-3', $5^{\prime}$-diaminobenzophe nones (ADBP-9, 10, 11, 13, 14) were synthesized according to the procedure described above (yield $43-53 \%$ ). Melting point (mp), ${ }^{1} \mathrm{H} \mathrm{NMR}$, IR, and elemental analysis are as follows.

\section{3,5-Dinitro-4'-nonylbenzophenone (ADBP-9)}

Mp $61.0-62.3^{\circ} \mathrm{C} ;{ }^{1} \mathrm{H}$ NMR (DMSO- $d_{6}$ ): $\delta 0.85(\mathrm{t}, 3 \mathrm{H},-$ $\left.\mathrm{Ar}-\mathrm{CH}_{2}-\mathrm{CH}_{2}-\left(\mathrm{CH}_{2}\right)_{9}-\mathrm{CH}_{3}, J=6.59 \mathrm{~Hz}\right), 1.24(\mathrm{~m}, 12 \mathrm{H}$, $\left.-\mathrm{Ar}-\mathrm{CH}_{2}-\mathrm{CH}_{2}-\left(\mathrm{CH}_{2}\right)_{6}-\mathrm{CH}_{3}\right), 1.59\left(\mathrm{~m}, 2 \mathrm{H},-\mathrm{Ar}-\mathrm{CH}_{2}-\mathrm{CH}_{2}-\right.$ $\left.\left(\mathrm{CH}_{2}\right)_{6}-\mathrm{CH}_{3}\right), 2.65\left(\mathrm{t}, 2 \mathrm{H},-\mathrm{Ar}-\mathrm{CH}_{2}-\mathrm{CH}_{2}-\left(\mathrm{CH}_{2}\right)_{6}-\mathrm{CH}_{3}, J=\right.$ $7.59 \mathrm{~Hz}), 5.00\left(\mathrm{~s}, 4 \mathrm{H}, \mathrm{NH}_{2}\right), 6.05(\mathrm{t}, 1 \mathrm{H}$, para to $\mathrm{C}=\mathrm{O}, J$ $=1.98 \mathrm{~Hz}), 6.13(\mathrm{~d}, 2 \mathrm{H}$, ortho to $\mathrm{C}=\mathrm{O}, J=1.98 \mathrm{~Hz}), 7.32$ (d, $2 \mathrm{H}$, ortho to $n$-nonyl group, $J=8.25 \mathrm{~Hz}$ ), $7.61(\mathrm{~d}, 2 \mathrm{H}$, meta to $n$-nonyl group, $J=8.25 \mathrm{~Hz}$ ) ppm. IR ( $\mathrm{KBr}$ ): 3340 $\left(\mathrm{NH}_{2}\right), 1640(\mathrm{C}=\mathrm{O}) \mathrm{cm}^{-1}$. Anal. Calcd for $\mathrm{C}_{22} \mathrm{H}_{30} \mathrm{~N}_{2} \mathrm{O}: \mathrm{C}$, $78.06 \%$;, $8.93 \%$; N, 8.28\%. Found: C, 77.99\%; H, $8.82 \% ; \mathrm{N}, 8.15 \%$.

\section{3,5-Dinitro-4'-decylbenzophenone (ADBP-10)}

Mp $61.3-62.9^{\circ} \mathrm{C} ;{ }^{1} \mathrm{H}$ NMR (DMSO- $\left.d_{6}\right): \delta 0.85(\mathrm{t}, 3 \mathrm{H}$, $\left.-\mathrm{Ar}-\mathrm{CH}_{2}-\mathrm{CH}_{2}-\left(\mathrm{CH}_{2}\right)_{7}-\mathrm{CH}_{3}, J=6.59 \mathrm{~Hz}\right), 1.24(\mathrm{~m}, 14 \mathrm{H}$, $\left.-\mathrm{Ar}-\mathrm{CH}_{2}-\mathrm{CH}_{2}-\left(\mathrm{CH}_{2}\right)_{7}-\mathrm{CH}_{3}\right), 1.59\left(\mathrm{~m}, 2 \mathrm{H},-\mathrm{Ar}-\mathrm{CH}_{2}-\mathrm{CH}_{2}-\right.$ $\left.\left(\mathrm{CH}_{2}\right)_{7}-\mathrm{CH}_{3}\right), 2.65\left(\mathrm{t}, 2 \mathrm{H},-\mathrm{Ar}-\mathrm{CH}_{2}-\mathrm{CH}_{2}-\left(\mathrm{CH}_{2}\right)_{7}-\mathrm{CH}_{3}, J=\right.$ $7.60 \mathrm{~Hz}), 5.00\left(\mathrm{~s}, 4 \mathrm{H}, \mathrm{NH}_{2}\right), 6.07(\mathrm{t}, 1 \mathrm{H}$, para to $\mathrm{C}=\mathrm{O}, J$ $=1.98 \mathrm{~Hz}), 6.13(\mathrm{~d}, 2 \mathrm{H}$, ortho to $\mathrm{C}=\mathrm{O}, J=1.98 \mathrm{~Hz}), 7.32$ (d, $2 \mathrm{H}$, ortho to $n$-decyl group, $J=8.25 \mathrm{~Hz}$ ), $7.62(\mathrm{~d}, 2 \mathrm{H}$, meta to $n$-nonyl group, $J=8.25 \mathrm{~Hz}) \mathrm{ppm}$. IR $(\mathrm{KBr}): 3350$ $\left(\mathrm{NH}_{2}\right), 1640(\mathrm{C}=\mathrm{O}) \mathrm{cm}^{-1}$. Anal . Calcd for $\mathrm{C}_{23} \mathrm{H}_{32} \mathrm{~N}_{2} \mathrm{O}: \mathrm{C}$, $78.37 \%$; H, 9.15\%; N, 7.95\%. Found:C, 77.88\%; H, 8.91\%; $\mathrm{N}, 7.50 \%$.

\section{3,5-Dinitro-4'-undecylbenzophenone (ADBP-11)}

Mp 61.3-62.8 ${ }^{\circ}{ }^{1}{ }^{1} \mathrm{H}$ NMR (DMSO- $d_{6}$ ): $\delta 0.85(\mathrm{t}, 3 \mathrm{H},-$ $\left.\mathrm{Ar}-\mathrm{CH}_{2}-\mathrm{CH}_{2}-\left(\mathrm{CH}_{2}\right)_{8}-\mathrm{CH}_{3}, \quad J=6.60 \mathrm{~Hz}\right), 1.24(\mathrm{~m}, 16 \mathrm{H}$, $\left.-\mathrm{Ar}-\mathrm{CH}_{2}-\mathrm{CH}_{2}-\left(\mathrm{CH}_{2}\right)_{8}-\mathrm{CH}_{3}\right), 1.59\left(\mathrm{~m}, 2 \mathrm{H}\right.$. $-\mathrm{Ar}-\mathrm{CH}_{2}-\mathrm{CH}_{2}-$ $\left.\left(\mathrm{CH}_{2}\right)_{8}-\mathrm{CH}_{3}\right), 2.64\left(\mathrm{t}, 2 \mathrm{H},-\mathrm{Ar}-\mathrm{CH}_{2}-\mathrm{CH}_{2}-\left(\mathrm{CH}_{2}\right)_{8}-\mathrm{CH}_{3}, J=\right.$ $7.59 \mathrm{~Hz}), 5.01\left(\mathrm{~s}, 4 \mathrm{H}, \mathrm{NH}_{2}\right), 6.07(\mathrm{t}, 1 \mathrm{H}$, para to $\mathrm{C}=\mathrm{O}, J$ $=1.98 \mathrm{~Hz}), 6.14(\mathrm{~d}, 2 \mathrm{H}$, ortho to $\mathrm{C}=\mathrm{O}, J=1.98 \mathrm{~Hz}), 7.32$ (d, $2 \mathrm{H}$, ortho to $n$-undecyl group, $J=8.25 \mathrm{~Hz}$ ), 7.61 (d, $2 \mathrm{H}$, meta to $n$-undecyl group, $J=8.25 \mathrm{~Hz}$ ) ppm. IR (KBr): $3350\left(\mathrm{NH}_{2}\right), 1640(\mathrm{C}=\mathrm{O}) \mathrm{cm}^{-1}$. Anal. Calcd for $\mathrm{C}_{24} \mathrm{H}_{34} \mathrm{~N}_{2} \mathrm{O}: \mathrm{C}, 78.64 \% ; \mathrm{H}, 9.35 \% ; \mathrm{N}, 7.64 \%$. Found: $\mathrm{C}$, $78.33 \% ; \mathrm{H}, 9.18 \%$;, $7.50 \%$.

\section{3,5-Dinitro-4'-tridecylbenzophenone ( $A D B P-13)$}

Mp 71.5-72.9 ${ }^{\circ} \mathrm{C} ;{ }^{1} \mathrm{H}$ NMR (DMSO- $d_{6}$ ): $\delta 0.85(\mathrm{t}, 3 \mathrm{H}$, $\left.-\mathrm{Ar}-\mathrm{CH}_{2}-\mathrm{CH}_{2}-\left(\mathrm{CH}_{2}\right)_{10}-\mathrm{CH}_{3}, J=6.60 \mathrm{~Hz}\right), 1.24(\mathrm{~m}, 20 \mathrm{H}$, $\left.-\mathrm{Ar}-\mathrm{CH}_{2}-\mathrm{CH}_{2}-\left(\mathrm{CH}_{2}\right)_{10}-\mathrm{CH}_{3}\right), 1.59\left(\mathrm{~m}, 2 \mathrm{H} .-\mathrm{Ar}-\mathrm{CH}_{2}-\mathrm{CH}_{2}\right.$ $\left.-\left(\mathrm{CH}_{2}\right)_{6}-\mathrm{CH}_{3}\right), 2.65$ (t, 2H. $\mathrm{Ar}-\mathrm{CH}_{2}-\mathrm{CH}_{2}-\left(\mathrm{CH}_{2}\right)_{6}-\mathrm{CH}_{3}, \mathrm{~J}$ $=7.56 \mathrm{~Hz}), 5.01\left(\mathrm{~s}, 4 \mathrm{H}, \mathrm{NH}_{2}\right), 6.05(\mathrm{t}, 1 \mathrm{H}$, para to $\mathrm{C}=\mathrm{O}$, $J=1.98 \mathrm{~Hz}), 6.14(\mathrm{~d}, 2 \mathrm{H}$, ortho to $\mathrm{C}=\mathrm{O}, J=1.98 \mathrm{~Hz}$ ), 7.32 (d, $2 \mathrm{H}$, ortho to $n$-tridecyl group, $J=8.25 \mathrm{~Hz}$ ), 7.61 (d, $2 \mathrm{H}$, meta to $n$-tridecyl group, $J=8.25 \mathrm{~Hz}$ ). IR ( $\mathrm{KBr}$ ): $3320\left(\mathrm{NH}_{2}\right), 1630(\mathrm{C}=\mathrm{O}) \mathrm{cm}^{-1}$. Anal. Calcd for $\mathrm{C}_{26} \mathrm{H}_{34} \mathrm{~N}_{2}$ O: C, 79.14\%; H, 9.71\%; N, 7.10\%. Found: C, 78.95\%; H, $9.65 \% ; \mathrm{N}, 7.03 \%$.

\section{3,5-Dinitro-4'-tetradecylbenzophenone (ADBP-14)}

Mp 74.1-75.0 $0^{\circ}$; ${ }^{1} \mathrm{H}$ NMR (DMSO- $\left.d_{6}\right): \delta 0.85(\mathrm{t}, 3 \mathrm{H}$, $\left.-\mathrm{Ar}-\mathrm{CH}_{2}-\mathrm{CH}_{2}-\left(\mathrm{CH}_{2}\right)_{11}-\mathrm{CH}_{3}, J=6.60 \mathrm{~Hz}\right), 1.23(\mathrm{~m}, 12 \mathrm{H}$,
$\left.-\mathrm{Ar}-\mathrm{CH}_{2}-\mathrm{CH}_{2}-\left(\mathrm{CH}_{2}\right)_{11}-\mathrm{CH}_{3}\right), 1.59\left(\mathrm{~m}, 2 \mathrm{H},-\mathrm{Ar}-\mathrm{CH}_{2}-\mathrm{CH}_{2}\right.$ $\left.-\left(\mathrm{CH}_{2}\right)_{6}-\mathrm{CH}_{3}\right), 2.64\left(\mathrm{t}, 2 \mathrm{H},-\mathrm{Ar}-\mathrm{CH}_{2}-\mathrm{CH}_{2}-\left(\mathrm{CH}_{2}\right)_{11}-\mathrm{CH}_{3}, \boldsymbol{J}\right.$ $=7.56 \mathrm{~Hz}), 5.02\left(\mathrm{~s}, 4 \mathrm{H}, \mathrm{NH}_{2}\right), 6.05(\mathrm{t}, 1 \mathrm{H}$, para to $\mathrm{C}=\mathrm{O}$, $J=1.98 \mathrm{~Hz}), 6.14(\mathrm{~d}, 2 \mathrm{H}$, ortho to $\mathrm{C}=\mathrm{O}, J=1.98 \mathrm{~Hz})$, 7.32 (d, $2 \mathrm{H}$, ortho to tetradecyl group, $J=8.25 \mathrm{~Hz}$ ), 7.62 (d, $2 \mathrm{H}$, meta to tetradecyl group, $J=8.25 \mathrm{~Hz}$ ). IR ( $\mathrm{KBr}$ ): $3360\left(\mathrm{NH}_{2}\right), 1640(\mathrm{C}=\mathrm{O}) \mathrm{cm}^{-1}$. Anal. Calcd for $\mathrm{C}_{27} \mathrm{H}_{40} \mathrm{~N}_{2}$ O: C, $79.36 \%$; H, $9.87 \%$; N, 6.86\%. Found: C, 79.24\%; H, $9.78 \% ; \mathrm{N}, 6.57 \%$.

\section{Poly(amic acid) preparations}

As a typical example, to a $30 \mathrm{~mL}$ flask were added $0.689 \mathrm{~g}(2.13 \mathrm{mmol}) \mathrm{BTDA}$ and $0.813 \mathrm{~g}(2.13 \mathrm{mmol}) \mathrm{ADB}$ $\mathrm{P}-12$. The system was purged by nitrogen and NMP was added. Monomer concentration was kept at $20 \mathrm{wt} \%$. The mixture was stirred at $60^{\circ} \mathrm{C}$ under nitrogen for $12 \mathrm{~h}$ to allow viscosity to increase. The BTDA/ADBP-12 poly(amic acid) solution was diluted with NMP and polymer concentration was kept at $10 \mathrm{wt} \%$. About one-third portions of the mixture were poured into excess water or methanol. Precipitated poly(amic acid) was filtered, washed with excess methanol and dried at room temperature for 2 days. The residual two-third portions of the poly(amic acid) solution were used for the following polyimide preparation. ${ }^{1} \mathrm{H}$ NMR ( pyridine- $\left.d_{5}\right): \delta 0.87$ (broad s, $\left.3 \mathrm{H},-\mathrm{Ar}-\mathrm{CH}_{2}-\mathrm{CH}_{2}-\left(\mathrm{CH}_{2}\right)_{9}-\mathrm{CH}_{3}\right), 1.25(\mathrm{~m}, 18 \mathrm{H},-$ $\left.\mathrm{Ar}-\mathrm{CH}_{2}-\mathrm{CH}_{2}-\left(\mathrm{CH}_{2}\right)_{9}-\mathrm{CH}_{3}\right), 1.61$ (m, 2H. $-\mathrm{Ar}-\mathrm{CH}_{2}-\mathrm{CH}_{2}-$ $\left.\left(\mathrm{CH}_{2}\right)_{9}-\mathrm{CH}_{3}\right), 2.57$ (t, $2 \mathrm{H}$. $\left.-\mathrm{Ar}-\mathrm{CH}_{2}-\mathrm{CH}_{2}-\left(\mathrm{CH}_{2}\right)_{9}-\mathrm{CH}_{3}\right), 7.1$ $-9.3(\mathrm{~m}, 13 \mathrm{H}$, arom.), $12.2(\mathrm{~m}, 2 \mathrm{H},-\mathrm{NH}-)$ ppm. Detailed description of ${ }^{1} \mathrm{H}$ NMR spectra will be given in the next section. IR (KBr): $3500(\mathrm{O}-\mathrm{H}$ and $\mathrm{N}-\mathrm{H}), 1780,1710$, $1660(\mathrm{C}=\mathrm{O}) \mathrm{cm}^{-1}$. Anal. Calcd for $\mathrm{C}_{42} \mathrm{H}_{42} \mathrm{~N}_{2} \mathrm{O}_{8}: \mathrm{C}, 71$. $78 \% ; \mathrm{H}, 6.02 \%$; N, 3.99\%. Found: C, 72.66; H, 5.85\%; N, $4.25 \%$.

\section{Polyimide Preparations}

As a typical example, to $10 \mathrm{~g}$ of above $10 \mathrm{wt} \%$ BTDA/ ADBP-12 poly(amic acid) solution were added $0.562 \mathrm{~g}$ $(7.10 \mathrm{mmol})$ of pyridine and $0.581 \mathrm{~g}(5.69 \mathrm{mmol})$ of acetic anhydride. The system was purged by nitrogen and stirred at $120^{\circ} \mathrm{C}$ under nitrogen for $5 \mathrm{~h}$. Powdered polyimides were obtained by precipitation into excess methanol, filtered, and washed with a large amount of methanol, and dried at $100{ }^{\circ} \mathrm{C}$ for 1 day. ${ }^{1} \mathrm{H}$ NMR (pyridine- $d_{5}$ ): $\delta 0.86$ (broad s, $\left.3 \mathrm{H},-\mathrm{Ar}-\mathrm{CH}_{2}-\mathrm{CH}_{2}-\left(\mathrm{CH}_{2}\right)_{9}-\mathrm{CH}_{3}\right), 1.25(\mathrm{~m}$, $\left.18 \mathrm{H},-\mathrm{Ar}-\mathrm{CH}_{2}-\mathrm{CH}_{2}-\left(\mathrm{CH}_{2}\right)_{9}-\mathrm{CH}_{3}\right), 1.61\left(\mathrm{~m}, 2 \mathrm{H},-\mathrm{Ar}-\mathrm{CH}_{2}-\right.$ $\left.\mathrm{CH}_{2}-\left(\mathrm{CH}_{2}\right)_{9}-\mathrm{CH}_{3}\right), 2.62$ (t, $2 \mathrm{H},-\mathrm{Ar}-\mathrm{CH}_{2}-\mathrm{CH}_{2}-\left(\mathrm{CH}_{2}\right)_{9}-$ $\left.\mathrm{CH}_{3}\right), 7.1-9.3(\mathrm{~m}, 13 \mathrm{H}$, arom.) ppm. Detailed description of ${ }^{1} \mathrm{H}$ NMR spectra is given in the next section. IR (KBr): $1780,1740(\mathrm{C}=\mathrm{O}), 1360(\mathrm{C}-\mathrm{N}) \mathrm{cm}^{-1}$. Anal. Calcd for $\mathrm{C}_{42} \mathrm{H}_{38} \mathrm{~N}_{2} \mathrm{O}_{6}: \mathrm{C}, 75.66 \% ; \mathrm{H}, 5.74 \% ; \mathrm{N}, 4.20 \%$. Found: C, $74.39 \% ; \mathrm{H}, 5.97 \% ; \mathrm{N}, 4.58 \%$.

\section{RESULTS AND DISCUSSION}

\section{Monomer Synthesis}

The diamines, alkyldiaminobenzophenones (ADBP) were prepared via two steps using 3,5-dinitrobenzoyl chloride as a starting material. The synthetic route is illustrated in Scheme 1. The Friedel-Crafts reaction of 3,5-dinitrobenzoyl chloride with alkylbenzene catalyzed by aluminum chloride in nitrobenzene gave 3,5-dinitro4 -alkylbenzophenones in good yields. Nitrobenzene, the 


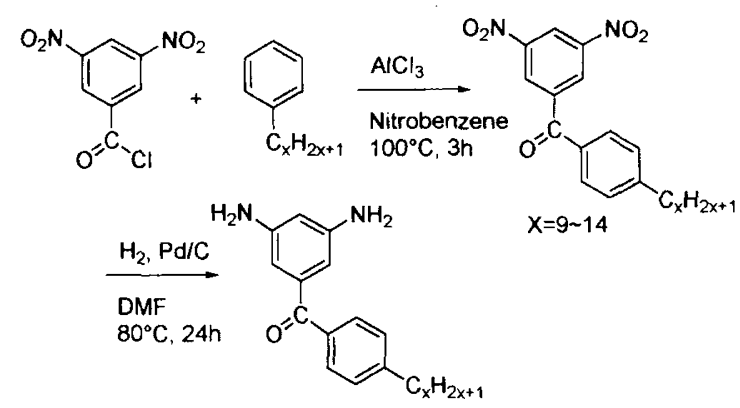

Scheme 1. Synthesis of alkyldiaminobenzophenon (ADBP).

most effective solvent to accelerate the reaction, was preferable in this Friedel-Crafts reaction. Other solvents such as dichoromethane and nitromethane gave the product in poor yield or complex mixtures.

The reduction of 3,5-dinitro-4'-alkylbenzophenone was performed by catalytic hydrogenation using palladium on carbon and hydrogen gas. Although hydrazine hydrate/ethanol system is sometime used for the reduction of nitrocompounds, this system is not preferable because the carbonyl group in 3,5-dinitro-4'-alkylbenzophenones reacts with hydrazine. Selective hydrogenation conditions for nitro groups are required. Otherwise the carbonyl group in 3,5-dinitro-4'-alkylbenzophenone is possibly converted to the alcohol and a methylene group. Thus, ADBP-9-14 were selectively obtained by mild catalytic hydrogenation using the laboratory balloon for introduction of hydrogen gas. Completion of the reduction can be judged by cessation of reduction of balloon volume. ADBP-9-14 were well characterized by ${ }^{1} \mathrm{H}$ NMR, IR and elemental analysis.

\section{Polymer Synthesis and Characterization}

The synthetic route of the soluble polyimides based on ADBP and various tetracarboxilic dianhydrides such as BTDA, TCA-AH, pyromellitic dianhydride (PMDA), $3,3^{\prime}, 4,4^{\prime}$-biphenyltetracarboxylic dianhydride (BPDA) is illustrated in Scheme 2. Two-step polymerization systems including poly(amic acid)s synthesis and chemical imidization were performed. The poly(amic acid)s unit based on BTDA and BPDA theoretically contain three isomers due to an unsymmetrical structure, while poly(amic acid)s unit based on PMDA or TCA-AH theoretically contain two or four isomers respectively. ${ }^{30,35}$ Poly(amic acis)s were obtained by reacting ADBP with equimolar amounts of tetracarboxylic dianhydrides at $60^{\circ} \mathrm{C}$ for $12 \mathrm{~h}$ under a nitrogen atmosphere. Polyimides were obtained by chemical imidization at $120^{\circ} \mathrm{C}$ in the presence of pyridine as base catalyst and acetic anhydride as dehydrating reagent. The synthetic conditions were previously optimized for TCA-AH based soluble polyimides in our laboratoly. ${ }^{30}$ The experimental results of various combinations of ADBP-9-14 and tetracarboxilic dianhydrides are summarized in Table I. All polymers were well characterized by ${ }^{1} \mathrm{H}$ NMR and IR. Carbon by elemental analysis was found lower than the calculated value and a trace amount of residue was observed after elemental analysis, probably due to the formation of char characteristic of heat resistant polymers. ${ }^{36}$

Out of 18 combinations studied, 9 soluble polyimides were obtained. In these cases, clear polyimide solutions
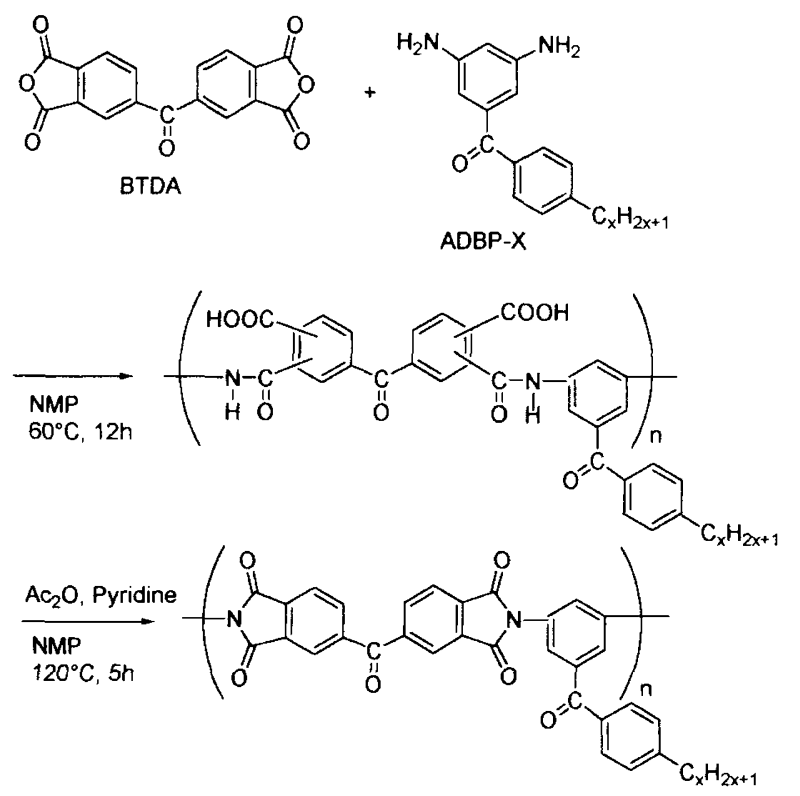

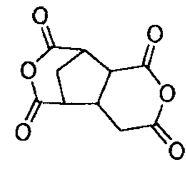

TCA-AH
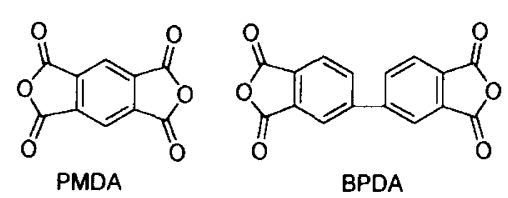

Scheme 2. Synthesis of soluble polyimides based on alkyldiaminobenzophenon (ADBP).

in NMP were eventually obtained. In other cases, clear polyamic acid solutions were obtained. However, gelation or precipitation took place during imidization process. The combinations of TCA-AH and ADBP-9-14 gave NMP soluble polyimides. TCA-AH having a polyalicyclic structure effective for the enhancement of solubility gives soluble polyimides regardless of diamine structure. ${ }^{30}$ Therefore, the effects of ADBP for the enhancement of solubility, were not confirmed in the above combinations. ADBP with even numbers of alkyl chains such as ADBP-10, 12, 14 gave NMP soluble polyimides in the combination of BTDA. As BTDA did not gave NMP soluble polyimides in the combinations of conventional aromatic diamine such as 1,4-phenylenediamine (PDA), $4,4^{\prime}$-diaminodiphenylmethane (DDM) and $\mathrm{DDE},{ }^{31}$ the effects of $\mathrm{ADBP}$ for the enhancement of solubility were confirmed. The polyimides based on BTDA and ADBP having odd numbers of alkyl chains such as ADBP-9, 11, 13 were not soluble in NMP. The odd-even dependence of physical properties is sometimes observed among polymers containing polymethylene units. For example, the odd-even dependence of phase transition parameters for main chain liquid crystalline polymers containing flexible spacers has been reported. ${ }^{37-42}$ Jin et al. reported that thermotropic main chain polyesters with even number polymethylene spacers are soluble only in a mixture of phenol and 1,1,2,2-tetrachloroethane (TCE), while those with odd numbers of methylene units were soluble in TCE. ${ }^{42}$ Solubility results of BTDA/ADBP polyimides are also of great interest because of unique odd-even effects of solubility in polymers with polymethylene units. It can be assumed that the conformation around $\mathrm{C}-\mathrm{C}$ bonds of the Iong chain linear alkyl groups and alignment of benzene ring attached with these alkyl 
Table I. Poly(amic acids) and corresponding polyimides using various combinations of tetracarboxylic dianhydrides and ADBP

\begin{tabular}{|c|c|c|c|c|c|c|c|}
\hline \multicolumn{2}{|c|}{ Monomer } & \multirow{4}{*}{$\begin{array}{l}\text { Polyamic acid } \\
\frac{\eta_{\text {inh }}{ }^{\mathrm{a}}}{\mathrm{dL} \mathrm{g}^{-1}}\end{array}$} & \multicolumn{5}{|c|}{ Polyimide } \\
\hline \multirow{3}{*}{\multicolumn{2}{|c|}{ Dianhydride Diamine }} & & \multirow{3}{*}{$\begin{array}{l}\text { Solubility } \\
\text { in NMP }\end{array}$} & \multirow{3}{*}{$\frac{\eta_{\text {inh }}{ }^{a}}{d L g}$} & \multirow{3}{*}{$\frac{\begin{array}{c}\text { Imidization } \\
\text { ratio }\end{array}}{\%}$} & \multicolumn{2}{|c|}{$\begin{array}{l}10 \% \text { Weight loss } \\
\text { temperature }\end{array}$} \\
\hline & & & & & & in Air & in $\mathrm{N}_{2}$ \\
\hline & & & & & & ${ }^{\circ} \mathrm{C}$ & ${ }^{\circ} \mathrm{C}$ \\
\hline TCA-AH & ADBP-9 & 0.25 & Soluble & 0.22 & 91 & 372 & 464 \\
\hline BTDA & & 0.37 & Insoluble & & & & \\
\hline TCA-AH & ADBP-10 & 0.27 & Soluble & 0.23 & 95 & 364 & 446 \\
\hline BTDA & & 0.22 & Soluble & 0.29 & 100 & 442 & 442 \\
\hline TCA-AH & ADBP-11 & 0.25 & Soluble & 0.24 & 92 & 353 & 456 \\
\hline BTDA & & 0.43 & Insoluble & & & & \\
\hline TCA-AH & ADBP-12 & 0.17 & Soluble & 0.22 & 100 & 376 & 458 \\
\hline BTDA & & 0.34 & Soluble & 0.37 & 100 & 468 & 464 \\
\hline BTDA & & 0.18 & Insoluble & & & & \\
\hline PMDA & & 0.23 & Insoluble & & & & \\
\hline TCA-AH & ADBP-13 & 0.15 & Soluble & 0.2 & 89 & 356 & 459 \\
\hline BTDA & & 0.34 & Insoluble & & & & \\
\hline BPDA & & 0.13 & Insoluble & & & & \\
\hline PMDA & & 0.18 & Insoluble & & & & \\
\hline TCA-AH & ADBP-14 & 0.19 & Soluble & 0.21 & 100 & 337 & 453 \\
\hline BTDA & & 0.35 & Soluble & 0.38 & 100 & 464 & 475 \\
\hline BPDA & & 0.18 & Insoluble & & & & \\
\hline PMDA & & 0.31 & Insoluble & & & & \\
\hline
\end{tabular}

groups and carbonyl group affect the solubility of these polyimides. The polyimides based on BPDA/ADBP and PMDA/ADBP were insoluble in NMP. These aromatic dianhydrides are less flexible compared to BTDA. ${ }^{31}$

High imidization ratios are required to obtain stable polyimides with excellent performance. ${ }^{43}$ Imidization ratios of polyimides are generally measured by IR measurement and comparing absorption intensities of amic acid carbonyl groups with those of imide carbonyl groups. ${ }^{44}$ In the case of soluble polyimides, NMR measurement is convenient because solution samples can be prepared that give more quantitative imidization ratio data. Figure 1 shows a representative ${ }^{1} \mathrm{H}$ NMR spectra of BTDA/ADBP poly(amic acid) and polyimide. Broad signals of $\mathrm{NH}$ protons of poly(amic acid) appear at 12.2 ppm, while the signals disappear in the corresponding polyimides. The imidization ratios of these polyimides were calculated from the reduction of intensity ratio of $\mathrm{NH}$ proton signals in poly(amic acid)s to the aromatic proton signals, and sufficiently high and in the range of $89-100 \%$ (Table I). The imidization ratios of polyimides based on TCA-AH tend to be slightly lower than the ones of aromatic soluble polyimides based on BTDA, and our previous paper showed similar tendencies. ${ }^{30}$ These tendencies are probably due to the fact that the kinked structure of TCA-AH and non-aromatic structure of TCA-AH slightly prevents the perfect chemical imidization.

The inherent viscosities of polyimides based on ADBP were low in the range of $0.21-0.38$, probably due to the lower reactivity of ADBP compared to conventional aromatic diamines such as DDE. As ADBP is a meta substituted diamine and has an electron withdrawing carbonyl group, the nucleophilicity of ADBP is considered to be lower than the aromatic para substituted diamines with electron donating groups such as ether groups. Although these ADBP polyimides show low inherent viscosities, solutions of these polyimides have film forming ability. The inherent viscosity of BTDA/ADBP polyimides is higher than that of TCA-AH/ADBP polyimides, probably due to the fact that BTDA is a highly reactive tetracarboxylic dianhydride with an electron withdrawing carbonyl group.

The thermal stability of these polyimides was evaluated by $10 \%$ weight loss temperature in TGA measurement. Ten percent weight loss temperatures of TCA-AH/ ADBP polyimides were in the range of $337-376^{\circ} \mathrm{C}$ in air and $446-464^{\circ} \mathrm{C}$ in nitrogen, while those of BTDA/ADBP polyimides were in the range of $442-468^{\circ} \mathrm{C}$ in air and $442-475^{\circ} \mathrm{C}$ in nitrogen. Although TCA-AH/ADBP polyimides which have aliphatic structures in tetracarboxylic dianhydride and diamine show lower thermal stability, BTDA/ADBP polyimides show higher thermal stability in spite that ADBP contains an aliphatic structure. In the case of TCA-AH/ADBP polyimides, $10 \%$ weight loss temperature in nitrogen was obviously higher than in air. Ten percent weight loss temperatures of BTDA/ ADBP polyimides showed similar values in air and nitrogen. The literature demonstrates that polymers with methyl and polymethylene groups show higher degradation temperature in air than in nitrogen due to the oxidation of these groups and gained weight. ${ }^{45-47}$ It may be presumed that the reason why TCA-AH/ADBP polyimides exhibited lower degradation temperature in air is that the main chain degradation due to TCA-AH units preferentially affects weight loss, even though the oxidation of long chain alkyl groups in ADBP units occurs simultaneously. 

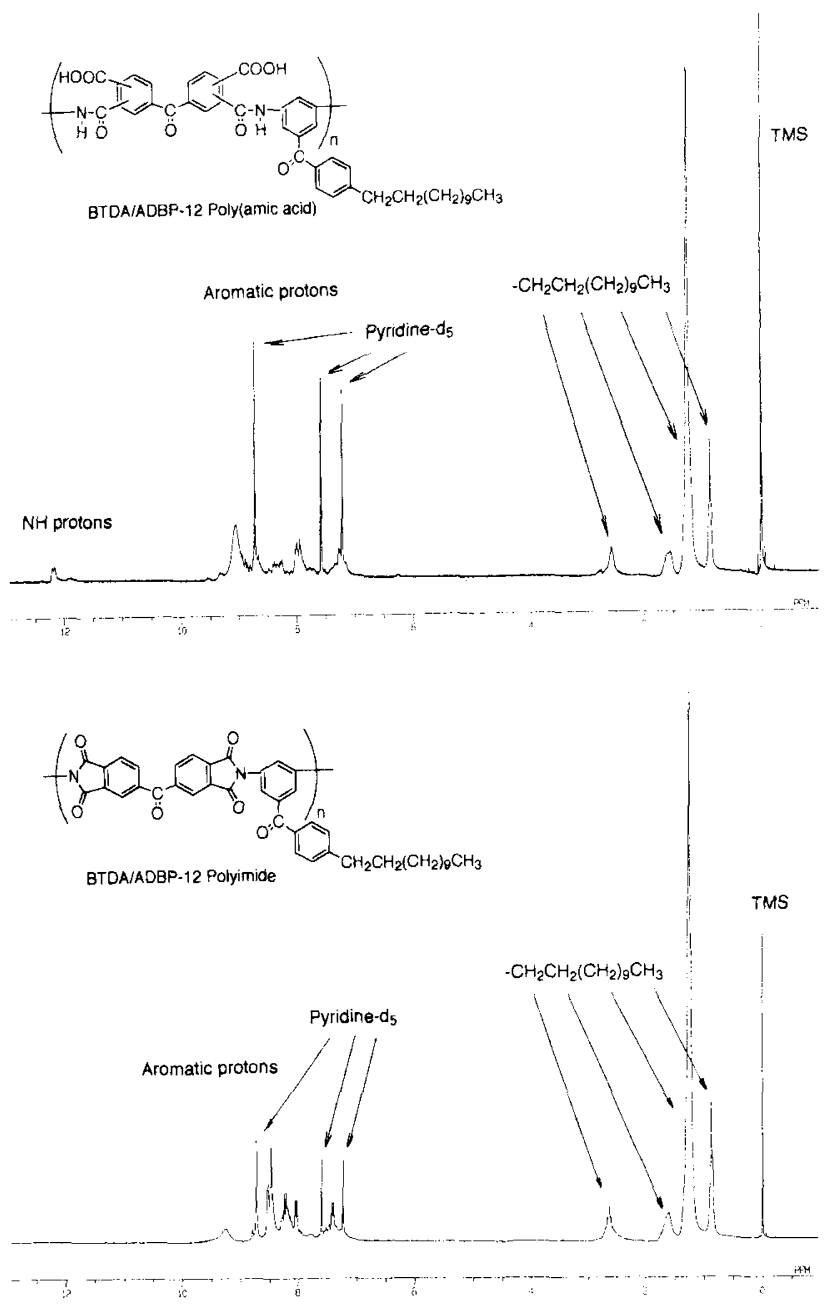

Figure 1. Representative ${ }^{1} \mathrm{H}$ NMR spectra of soluble poly(amic acid)s and polyimides based on alkyldiaminobenzophenon (BTDA) ADBP-12)

The experimental results of copolymerization based on BTDA/ADBP-12-14/DDE are summarized in Scheme 3 and Table II. BTDA/ADBP-12 and BTDA/ADBP-14 homopolyimides, and BTDA/ADBP-12/DDE copolyimides and BTDA/ADBP-12/DDE copolyimides containing 40 mol\% of ADBP or more were soluble in NMP. Incorporation of high reactive aromatic diamines, DDE results in increment of inherent viscosity of maximum value of

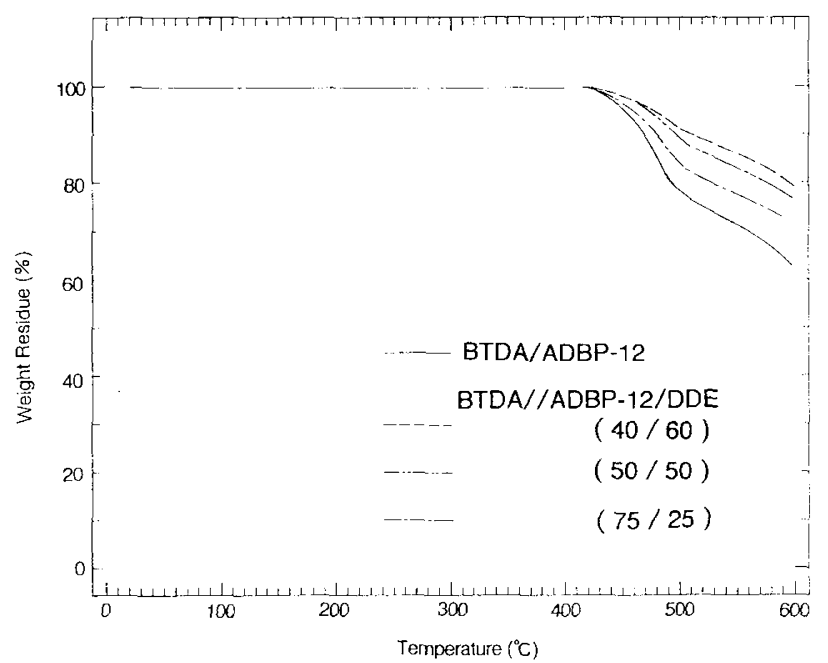

Figure 2. TGA curves of representative soluble polyimides and copolyimides based on alkyldiaminobenzophenone in nitrogen (BTDA/ADBP-12/DDE).

0.67 in BTDA/ADBP-14/DDE (100/40/60 mol\%). The experimental results of copolymerization based on BTDA/ ADBP-13/DDE are quite unique. Although BTDA/ ADBP-13 homopolyimide is insoluble, the copolymers, BTDA/ADBP-13/DDE (100/75/25) and BTDA/ADBP-13/ $\mathrm{DDE}(100 / 50 / 50)$ are soluble in NMP. The solubility of these copolyimides may be improved by randomizing effect based on copolymerization as well as entropy effect of long chain linear alkyl groups. Bryant reported that the copolyimides using only conventional monomers such as 4,4'-oxydiphthalic anhydride, BPDA, and 3,4'diaminodiphenyl ether are soluble at a selected monomer ratio by similar randomizing effect. ${ }^{48}$ As the incorporation of DDE resulted in the reduction of aliphatic components of polyimides, $10 \%$ weight loss temperatures of BTDA/ADBP-12-14/DDE copolyimides increased by increment of DDE component and the highest temperature reached $509^{\circ} \mathrm{C}$, which is near those of wholly aromatic polyimides (Figure 2, Table II).

The solubility of the obtained polyimides was determined in 9 common solvents at $5 \mathrm{wt} \%$ concentration (Table III). Homopolyimides containing ADBP were soluble in polar solvents such as NMP, DMF, DMAc, DMSO, DMI, $m$-cresol, THF, and $\mathrm{H}_{2} \mathrm{SO}_{4}$. Polyimides containing

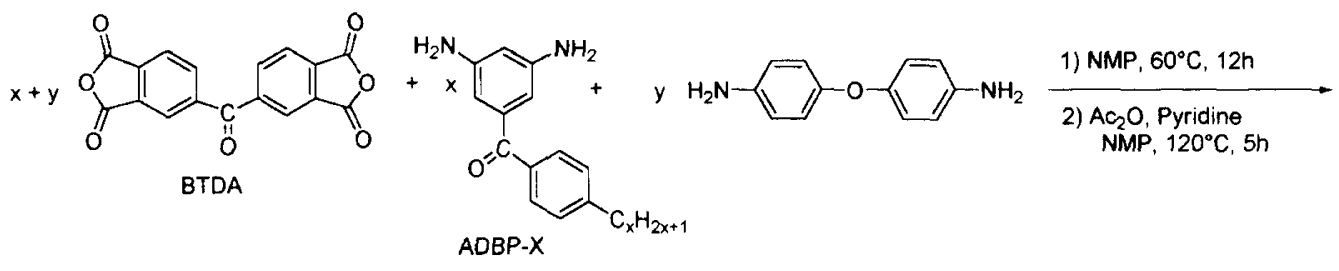

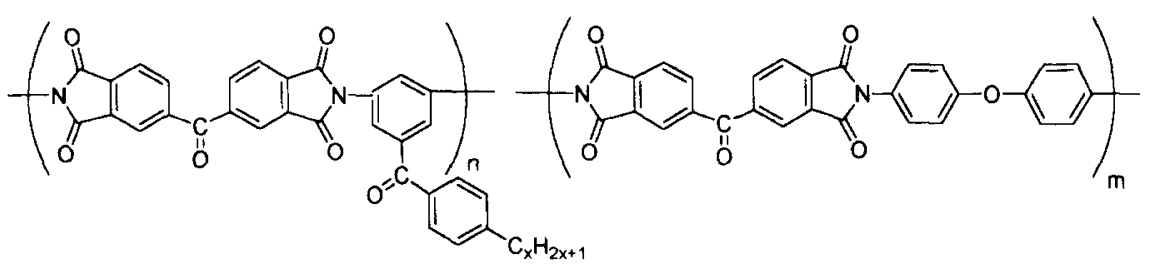

Scheme 3. Synthesis of soluble copolyimides based on alkyldiaminobenzophenon (ADBP). 
Table II. Copoly(amic acids) and corresponding copolyimides based on BTDA/ADBP-12-14/DDE

\begin{tabular}{|c|c|c|c|c|c|c|c|}
\hline \multirow{2}{*}{\multicolumn{2}{|c|}{$\begin{array}{l}\text { Diamine }^{\mathrm{a}} \\
\text { ADBP/DDE }\end{array}$}} & \multirow{4}{*}{$\begin{array}{c}\text { Polyamic acid } \\
\eta_{\text {inh }}{ }^{\mathrm{b}} \\
\mathrm{dL} \mathrm{g}^{-1}\end{array}$} & \multicolumn{5}{|c|}{ Polyimide } \\
\hline & & & \multirow{3}{*}{$\begin{array}{l}\text { Solubility } \\
\text { in NMP }\end{array}$} & \multirow{3}{*}{$\frac{\eta_{\text {inh }}{ }^{\mathrm{b}}}{\mathrm{dL} \mathrm{g}^{-1}}$} & \multirow{3}{*}{$\frac{\begin{array}{c}\text { Imidization } \\
\text { ratio }^{c}\end{array}}{\%}$} & \multicolumn{2}{|c|}{$\begin{array}{l}10 \% \text { Weight loss } \\
\text { temperature }^{\mathrm{d}}\end{array}$} \\
\hline $\mathrm{x}$ & $\mathrm{y}$ & & & & & in Air & in $\mathrm{N}_{2}$ \\
\hline $\mathrm{mol} \%$ & mol\% & & & & & ${ }^{\circ} \mathrm{C}$ & ${ }^{\circ} \mathrm{C}$ \\
\hline \multicolumn{8}{|c|}{ ADBP-12 } \\
\hline 0 & 100 & 0.68 & Insoluble & & & & \\
\hline 25 & 75 & 0.44 & Insoluble & & & & \\
\hline 40 & 60 & 0.46 & Soluble & 0.46 & 100 & 477 & 509 \\
\hline 50 & 50 & 0.49 & Soluble & 0.37 & 100 & 467 & 500 \\
\hline 75 & 25 & 0.49 & Soluble & 0.46 & 100 & 469 & 481 \\
\hline 100 & 0 & 0.34 & Soluble & 0.37 & 100 & 468 & 464 \\
\hline \multicolumn{8}{|c|}{ ADBP-13 } \\
\hline 0 & 100 & 0.68 & Insoluble & & & & \\
\hline 25 & 75 & 0.36 & Insoluble & & & & \\
\hline 40 & 60 & 0.31 & Insoluble & & & & \\
\hline 50 & 50 & 0.43 & Soluble & 0.39 & 100 & 479 & 485 \\
\hline 75 & 25 & 0.38 & Soluble & 0.24 & 100 & 482 & 479 \\
\hline 100 & 0 & 0.22 & Insoluble & & & & \\
\hline \multicolumn{8}{|c|}{ ADBP-14 } \\
\hline 0 & 100 & 0.68 & Insoluble & & & & \\
\hline 25 & 75 & 0.44 & Insoluble & & & & \\
\hline 40 & 60 & 0.41 & Soluble & 0.67 & 100 & 480 & 499 \\
\hline 50 & 50 & 0.34 & Soluble & 0.64 & 100 & 480 & 491 \\
\hline 75 & 25 & 0.29 & Soluble & 0.48 & 100 & 474 & 478 \\
\hline 100 & 0 & 0.35 & Soluble & 0.38 & 100 & 464 & 475 \\
\hline
\end{tabular}

Table III. Solubility behavior of polyimides and copolyimides based on ADBP in various solvents

\begin{tabular}{|c|c|c|c|c|c|c|c|c|c|c|}
\hline \multirow{2}{*}{\multicolumn{2}{|c|}{$\frac{\text { Polyimides or Copolyimide }}{\text { Monomer composition }}$}} & \multicolumn{9}{|c|}{ Solubility $^{a}$} \\
\hline & & \multirow{2}{*}{ NMP } & \multirow{2}{*}{ DMF } & \multirow{2}{*}{ DMAc } & \multirow{2}{*}{ DMSO } & \multirow{2}{*}{ DMI } & \multirow{2}{*}{$m$-Cresol } & \multirow{2}{*}{ THF } & \multirow{2}{*}{$\mathrm{CH}_{2} \mathrm{Cl}_{2}$} & \multirow{2}{*}{$\mathrm{H}_{2} \mathrm{SO}_{4}$} \\
\hline Dianhydride & Diamine/mol\% & & & & & & & & & \\
\hline \multicolumn{11}{|l|}{ Polyimides } \\
\hline TCA-AH & ADBP-9 & $\mathrm{S}$ & $\mathrm{S}$ & $\mathrm{S}$ & $\mathrm{S}$ & $\mathrm{S}$ & $\mathrm{S}$ & $\mathrm{S}$ & I & $\mathrm{S}$ \\
\hline TCA-AH & ADBP-10 & $\mathrm{S}$ & $\mathrm{S}$ & $\mathrm{S}$ & $\mathrm{S}$ & $\mathrm{S}$ & $\mathrm{S}$ & $\mathrm{S}$ & I & $\mathrm{S}$ \\
\hline BTDA & ADBP-10 & $\mathrm{S}$ & $\mathrm{S}(\mathrm{h})$ & $\mathrm{S}$ & $\mathrm{S}$ & $\mathrm{S}$ & $\mathrm{S}$ & $\mathrm{S}$ & $\mathrm{I}$ & $\mathrm{S}$ \\
\hline TCA-AH & ADBP-11 & $\mathrm{S}$ & $\mathrm{S}$ & $\mathrm{S}$ & $\mathrm{S}$ & $\mathrm{S}$ & $\mathrm{S}$ & $\mathrm{S}$ & $\mathrm{S}$ & $\mathrm{S}$ \\
\hline TCA-AH & ADBP-12 & $\mathrm{S}$ & $\mathrm{S}$ & $\mathrm{S}$ & $\mathrm{S}$ & $\mathrm{S}$ & $\mathrm{S}$ & $\mathrm{S}$ & $\mathrm{S}$ & $\mathrm{S}$ \\
\hline BTDA & ADBP-12 & $\mathrm{S}$ & $\mathrm{S}$ & $\mathrm{S}$ & $\mathrm{S}(\mathrm{h})$ & $\mathrm{S}$ & $\mathrm{S}$ & $\mathrm{S}$ & $\mathrm{S}$ & $\mathrm{S}$ \\
\hline TCA-AH & ADBP-13 & $\mathrm{S}$ & $\mathrm{S}$ & $\mathrm{S}$ & $\mathrm{S}$ & $\mathrm{S}$ & $\mathrm{S}$ & $\mathrm{S}$ & $\mathrm{S}$ & $\mathrm{S}$ \\
\hline TCA-AH & ADBP-14 & $\mathrm{S}$ & $\mathrm{S}$ & $\mathrm{S}$ & $\mathrm{S}$ & $\mathrm{S}$ & $\mathrm{S}$ & $\mathrm{S}$ & $\mathrm{I}$ & $\mathrm{S}$ \\
\hline BTDA & ADBP-14 & $\mathbf{S}$ & $\mathrm{S}(\mathrm{h})$ & $\mathrm{S}(\mathrm{h})$ & $\mathrm{S}(\mathrm{h})$ & $\mathrm{S}$ & $\mathrm{S}$ & $\mathrm{S}$ & I & $\mathrm{S}$ \\
\hline \multicolumn{11}{|l|}{ Copolyimides } \\
\hline BTDA & ADBP-12(40) DDE $(60)$ & $\mathrm{S}$ & $\mathrm{S}(\mathrm{h})$ & $\mathrm{S}$ & $\mathrm{S}(\mathrm{h})$ & $\mathrm{S}$ & $\mathrm{S}$ & I & $\mathrm{I}$ & $\mathrm{S}$ \\
\hline BTDA & ADBP-12(50) DDE $(50)$ & $\mathrm{S}$ & $\mathrm{S}(\mathrm{h})$ & $\mathrm{S}$ & $\mathrm{S}(\mathrm{h})$ & $\mathrm{S}$ & $\mathrm{S}$ & I & $\mathrm{I}$ & $\mathrm{S}$ \\
\hline BTDA & ADBP-12(75) DDE(25) & $\mathrm{S}$ & $\mathrm{S}(\mathrm{h})$ & $\mathrm{S}$ & $\mathrm{S}(\mathrm{h})$ & $\mathrm{S}$ & $\mathrm{S}$ & $\mathrm{I}$ & $\mathrm{I}$ & $\mathrm{S}$ \\
\hline BTDA & ADBP-13(50) DDE $(50)$ & $\mathrm{S}$ & I & $\mathrm{S}$ & $\mathrm{S}(\mathrm{h})$ & $\mathrm{S}$ & $\mathrm{S}$ & $\mathrm{I}$ & I & $\mathrm{S}$ \\
\hline BTDA & ADBP-13(75) DDE(25) & $\mathrm{S}$ & $\mathrm{S}(\mathrm{h})$ & $\mathrm{S}(\mathrm{h})$ & $\mathrm{S}(\mathrm{h})$ & $\mathrm{S}$ & $\mathrm{S}$ & $\mathrm{I}$ & $\mathrm{I}$ & $\mathrm{S}$ \\
\hline BTDA & ADBP-14(40) DDE $(60)$ & $\mathrm{S}$ & $\mathrm{S}$ & $\mathrm{S}$ & $\mathrm{S}(\mathrm{h})$ & $\mathrm{S}$ & $\mathrm{S}$ & $\mathrm{I}$ & $\mathrm{I}$ & $\mathrm{S}$ \\
\hline BTDA & ADBP-14(50) DDE $(50)$ & $\mathrm{S}$ & $\mathrm{S}(\mathrm{h})$ & $\mathrm{S}$ & $\mathrm{S}(\mathrm{h})$ & $\mathrm{S}$ & $\mathrm{S}$ & $\mathrm{I}$ & $\mathrm{I}$ & $\mathrm{S}$ \\
\hline BTDA & ADBP-14(75) DDE(25) & $\mathrm{S}$ & $\mathrm{S}(\mathrm{h})$ & $\mathrm{S}$ & $\mathrm{S}(\mathrm{h})$ & $\mathrm{S}$ & $\mathrm{S}$ & $\mathrm{I}$ & $\mathrm{I}$ & $\mathrm{S}$ \\
\hline
\end{tabular}

${ }^{a} \mathrm{~S}$, soluble; $\mathrm{S}(\mathrm{h})$, soluble at temperature heated; I, insoluble.

ADBP with 11-13 methylene units were soluble in $\mathrm{CH}_{2} \mathrm{Cl}_{2}$. The copolyimides based on BTDA/ADBP$11-13 / \mathrm{DDE}$ were less soluble in comparison with homopolyimides. These copolyimides were soluble in NMP, DMAc, DMF, DMSO, DMI, $m$-cresol, and $\mathrm{H}_{2} \mathrm{SO}_{4}$, but, insoluble in THF and $\mathrm{CH}_{2} \mathrm{Cl}_{2}$. Solubility in DMF and DMAc suggests that copolyimides with odd number ADBP (ADBP-13) are less soluble than with even number ADBP (ADBP-12 and ADBP-14), and solubility de- creases with increase of DDE.

\section{CONCLUSION}

Novel polyimides and copolyimides based on alkyldiaminobenzophenone (ADBP) having long chain linear alkyl groups and a conventional tetracarboxylic dianhydride, BTDA were successfully synthesized and showed excellent solubility in various polar solvents and ther- 
mal stability. This solubility is assumed to be result from the entropy effect of flexible alkyl groups. ADBP with even numbers of alkyl groups were found more effective than those with odd numbers probably due to the conformational effect of long chain linear alkyl groups. The copolymerization using a conventional aromatic diamine, that was DDE resulted in improvement of the molecular weight and thermal stability.

Acknowledgments. The authors thank Dr. Yoshiyuki Oishi of Iwate University, Dr. Atsushi Takahara of Kyushu University and Dr. Virgil Percec of University of Pennsylvania for valuable advise. Financial support from Technopolis Foundation of Kurume-Tosu is gratefully acknowledged.

\section{REFERENCES}

1. (a) K. L. Mittal, Ed., "Polyimides," Plenum Press, New York, N.Y., 1984; (b) M. K. Ghosh and K. L. Mittal, Ed., "Polyimides," Marcel Dekker, New York, N.Y., 1996.

2. J. E. McGrath, M. E. Rogers, C. A. Arnold, Y. J. Kim, and J. C. Hedrick, Macromol. Chem., Macromol. Symp. 51, 103 (1991).

3. S. Z. D. Cheng, F. E. Arnold, Jr., A. Zhang, S. L.-C. Hsu, and F. W. Harris, Macromolecules, 24, 5856 (1991).

4. F. E. Arnold, Jr., S. Z. D. Cheng, S. L.-C. Hsu, C. J. Lee, F. W. Harris, and S.-F. Lau, Polymer, 33, 5179 (1992).

5. M. E. Rogers, M. H. Brink, J. E. McGrath, and A. Brennan, Polymer, 34, 849 (1993).

6. J. W. Park, M. Lee, M.-H. Lee, J. W. Liu, S. D. Kim, J. Y. Chang, and S. B. Rhee, Macromolecules, 27, 3459 (1994).

7. S.-H. Lin, F. Li, S. Z. D. Cheng, and F. W. Harris, Macromolecules, 31, 2080 (1998).

8. P. A. Falcigno, S. Jasne, and M. King, J. Polym. Sci., Polym. Chem. Ed., 30, 1433 (1992).

9. C.-P. Yang and J.-H. Lin, J. Polym. Sci., Polym. Chem. Ed., 32, 423 (1994).

10. D.-J. Liaw and B.-Y. Liaw, J. Polym. Sci., Polym. Chem. Ed., 35, 1527 (1997).

11. D. J. Liaw and B. Y. Liaw, Polymer, 40, 3183 (1999).

12. Y. Oishi, M. Ishida, M. Kakimoto, Y. Imai, and T. Kurosaki, J. Polym. Sci., Polym. Chem. Ed., 30, 1027 (1992).

13. I. K. Spiliopoulos and J. A. Mikroyannidis, Macromolecules, 31, 1236 (1998).

14. I. K. Spiliopoulos, J. A. Mikroyannidis, and G. M. Tsivgoulis, Macromolecules, 31, 522 (1998).

15. X. Sun, Y.-K. Yang, and F. Lu, Macromolecules, 31, 4291 (1998).

16. J. K. Kallitsis, K. G. Gravalos, A. Hilberer, and G. Hadzilioannou, Macromolecules, 30, 2989 (1997).

17. J. A. Mikroyannidis, Macromolecules, 28, 5177 (1995).

18. C. A. Arnold, J. D. Summers, Y. P. Chen, R. H. Bott, D. Chen, and J. E. McGrath, Polymer, 30, 986 (1989).

19. N. Furukawa, Y. Yamada, M. Furukawa, M. Yuasa, and Y. Kimura, J. Polym.Sci., Polym. Chem. Ed., 35, 2239 (1997).
20. S. Itamura, M. Yamada, S. Tamura, T. Matsumoto, and T. Kurosaki, Macromolecules, 26, 3940 (1993).

21. M. Yamada, M. Kusama, T. Matsumoto, and T. Kurosaki, Macromolecules, 26, 4961 (1993).

22. M. Kusama, T. Matsumoto, and T. Kurosaki, Macromolecules, 27, 1117 (1994).

23. Y.-T. Chern, Macromolecules, 31, 1898 (1998).

24. D. J. Liaw and B. Y. Liaw, Macromol. Chem. Phys., 200, 1326 (1999).

25. D. J. Liaw, B. Y. Liaw, and C. Y. Chung, J. Polym. Sci., Polym. Chem. Ed., 37, 2815 (1999).

26. D. Sek, A. Wanic, and E. Shab-Balcerzak, J. Polym.Sci., Polym. Chem. Ed., 35, 539 (1997).

27. S.-H. Hsiao, C.-P. Yang, and C.-Y. Yang, J. Polym. Sci., Polym. Chem. Ed., 35, 1487 (1997).

28. J. A. Mikroyannidis, J. Polym. Sci., Polym. Chem. Ed., 35, 1353 (1997).

29. D. J. Liaw, B. Y. Liaw, L. J. Li, B. Sillion, R. Mercier, R. Thiria, and H. Sekiguchi, Chem. Mater., 10, 734 (1998).

30. Y. Tsuda, Y. Tanaka, K. Kamata, N. Hiyoshi, S. Mataka, Y. Matsuki, M. Nishikawa, S. Kawamura, and N. Bessho, Polym. J., 29, 574 (1997).

31. Y. Tsuda, K. Etou, N. Hiyoshi, M. Nishikawa, Y. Matsuki, and N. Bessho, Polym. J., 30, 222 (1998).

32. K.-W. Lee, S.-H. Paek, A. Lien, C. Durning and H. Fukuro, Macromolecules, 29, 8894 (1996).

33. H. Wang, Z. Shen, M. Guo, S. Z. D. Cheng, and F. W. Harris, Polym. Prepr., Am. Chem. Soc., Div. Polym. Chem., 40, 884 (1999).

34. Y. Tsuda, Polym. Prepr., Am. Chem. Soc., Div. Polym. Chem., 40(2), 1215 (1999).

35. V. M. Denisov, V. M. Svetlichnyi, A. Gindin, V. A. Zubkov, A. I. Kol'tsov, M. M. Koton, and V. V. Kudryavtsev, Polym. Sci. USSR, A 21, 1644 (1979).

36. Y. Oishi, M. Ishida, H. Takado, M. Yoneyama, M. Kakimoto, and Y. Imai, J. Polym. Sci., Polym. Chem. Ed., 28, 1763 (1990).

37. R. B. Blumstein and A. Blumstein, Mol. Cryst. Liq. Cryst., 165, 361 (1988).

38. A. Blumstein and O. Thomas, Macromolecules, 15, 1264 (1982).

39. A. Blumstein, Polym. J., 17, 277 (1985).

40. R. S. Kumar, S. B. Clough, and A. Blumstein, Mol. Cryst. Liq. Cryst., 157, 387 (1988).

41. V. Percec and Y. Tsuda, Macromolecules, 23, 3509 (1990).

42. J.-Il Jin, E-J. Choi, S.-C. Ryu, and R. W. Lenz, Polym. J., 18, 63 (1986).

43. M. Nishikawa, Y. Yokoyama, N. Bessho, D. -S. Seo, Y. Iimura, and S. Kobayashi, Jpn. J. Appl. Phys., 33, L 810 (1994).

44. S. Numata, K. Fujisaki, and N. Kinjo, in "Polyimides," Plenum Press, New York, N.Y., 1984, p 259.

45. D. J. Liaw, B. Y. Liaw, J. R. Chen, and C. M. Yang, Macromolecules, 32, 6860 (1999).

46. F. M. Rugg, J. J. Smith, and R. C. Bacon, J. Polym. Sci., 13, 535 (1954)

47. J. P. Luongo, J. Polym. Sci., 42, 139 (1960).

48. R. G. Bryant, Polym. Prepr., Am. Chem. Soc., Div. Polym. Chem., 35, 517 (1994). 